\title{
The value of blood lactate kinetics in critically ill patients: a systematic review
}

\author{
Jean-Louis Vincent ${ }^{*}$, Amanda Quintairos e Silva ${ }^{\dagger}$, Lúcio Couto $\mathrm{Jr}^{\dagger}$ and Fabio S. Taccone
}

\begin{abstract}
Background: The time course of blood lactate levels could be helpful to assess a patient's response to therapy. Although the focus of published studies has been largely on septic patients, many other studies have reported serial blood lactate levels in different groups of acutely ill patients.

Methods: We performed a systematic search of PubMed, Science Direct, and Embase until the end of February 2016 plus reference lists of relevant publications. We selected all observational and interventional studies that evaluated the capacity of serial blood lactate concentrations to predict outcome. There was no restriction based on language. We excluded studies in pediatric populations, experimental studies, and studies that did not report changes in lactate values or all-cause mortality rates. We separated studies according to the type of patients included. We collected data on the number of patients, timing of lactate measurements, minimum lactate level needed for inclusion if present, and suggested time interval for predictive use.

Results: A total of 96 studies met our criteria: 14 in general ICU populations, five in general surgical ICU populations, five in patients post cardiac surgery, 14 in trauma patients, 39 in patients with sepsis, four in patients with cardiogenic shock, eight in patients after cardiac arrest, three in patients with respiratory failure, and four in other conditions. A decrease in lactate levels over time was consistently associated with lower mortality rates in all subgroups of patients. Most studies reported changes over 6, 12 or 24 hrs, fewer used shorter time intervals. Lactate kinetics did not appear very different in patients with sepsis and other types of patients. A few studies suggested that therapy could be guided by these measurements.

Conclusions: The observation of a better outcome associated with decreasing blood lactate concentrations was consistent throughout the clinical studies, and was not limited to septic patients. In all groups, the changes are relatively slow, so that lactate measurements every 1-2 hrs are probably sufficient in most acute conditions. The value of lactate kinetics appears to be valid regardless of the initial value.
\end{abstract}

\section{Background}

Since the early studies by Weil and others [1-3], blood lactate concentrations have been used widely as a marker of altered tissue perfusion in critically ill patients [4]. In physiological conditions, about $1500 \mathrm{mmol}$ of lactate is produced daily from various organs, including the muscle, the intestine, the red blood cells, the brain, and the skin [5]. Lactate is metabolized by the liver (about $60 \%$ ), the kidneys (about $30 \%$ ), and other organs [5]. The normal blood lactate concentration is around $1 \mathrm{mEq} / \mathrm{l}[6]$. Even minor increases in lactate concentrations

\footnotetext{
* Correspondence: jlvincent@intensive.org

${ }^{\dagger}$ Equal contributors

Department of Intensive Care, Erasme Hospital, Université Libre de Bruxelles, Route de Lennik 808, 1070 Brussels, Belgium
}

to $>1.5 \mathrm{mEq} / \mathrm{l}$ are associated with higher mortality rates $[6,7]$. The exact pathophysiologic mechanisms of hyperlactatemia have been much debated, because the condition does not always simply reflect the development of anaerobic metabolism [8]. In sepsis in particular, metabolic alterations can contribute to elevated blood lactate concentrations, including increased glycolysis, catecholaminestimulated $\mathrm{Na}-\mathrm{K}$ pump activity, alterations in pyruvate dehydrogenase activity, and reduced lactate clearance primarily as a result of liver hypoperfusion. Regardless of these mechanisms, hyperlactatemia is a hallmark characteristic of shock states $[4,9]$ and the degree of increase in lactate concentrations is directly related to the severity of the shock state and to mortality rates $[10,11]$. 
As for the blood levels of any substance, elevated lactate levels can be the result of increased production, reduced elimination, or both. A dynamic evaluation of serial lactate concentrations may thus be more informative than a single value. This concept of repeating blood lactate concentrations over time as an indicator of response to therapy was first proposed in 1983 [12], based on an idea raised after a publication by Orringer et al. in 1977 [13] showing that the decrease in lactate levels after cessation of grand mal seizures was actually quite rapid, with a half-life of about $50 \%$ in $1 \mathrm{hr}$. Many studies have since emphasized that changes in lactate over the first hrs of treatment may represent a valuable monitoring tool. Some studies have even proposed integrating changes in lactate concentrations as a target in therapeutic protocols [14-17] or including them as one of the sepsis resuscitation "bundles" [18]. A number of investigators have used the term "lactate clearance" to describe decreasing lactate levels, but this is incorrect for two reasons. The first is that the changes in lactate concentrations over time reflect changes in production and in elimination. The decrease in lactate over time may reflect decreased (over)production more than increased clearance by the liver and other organs $[19,20]$. The specific study of lactate clearance would require intravenous injection of radiolabeled lactate, as has been done in several studies [21, 22]. The second reason why use of the term is incorrect is that "clearance" or "elimination" implies a progressive normalization of blood lactate concentrations, which is too simplistic. Blood lactate concentrations can have a complex evolution and may even increase over time (Fig. 1), a situation that one should then call "negative lactate clearance".

We performed a literature search on this subject to address several questions. First, is the observation of a better prognosis with decreasing lactate concentrations a consistent finding in all types of critically ill patient? Second, although some studies have suggested that repeated lactate measurements may be particularly useful in sepsis, can similar observations be made in other acute disease states or even in heterogeneous groups of critically ill patients? Third, how fast should lactate concentrations decrease in optimal conditions and is there any particular time interval that could be recommended? Fourth, some studies in emergency medicine considered only patients with lactate values $>4 \mathrm{mEq} / \mathrm{l}$ as an at-risk population, but is this approach valid? In other words, is the study of lactate kinetics more useful when lactate concentrations exceed a given value?

\section{Methods}

We searched databases of PubMed, Science Direct, and Embase until the end of February 2016 to identify studies that evaluated the capacity of serial blood lactate concentrations to predict outcome, using the search terms "Lactate levels" OR "lactate clearance" AND "shock" OR "critically ill" AND "mortality". We included original prospective or retrospective clinical studies. There was no restriction based on language. We excluded studies in pediatric populations, experimental studies, case reports, and studies that did not report changes over time in lactate values or relationship of changes in lactate concentrations to all-cause mortality rates. We had no restriction on the initial location in the hospital (e.g., ICU, trauma unit, emergency room, operating room). We also checked the reference lists of included articles to capture any references missed during the search. We classified the different adult populations into general ICU patients, general surgical ICU patients, cardiac surgery patients, trauma patients, patients with sepsis, patients with cardiogenic shock, post-cardiac arrest patients, patients with respiratory failure, and others.

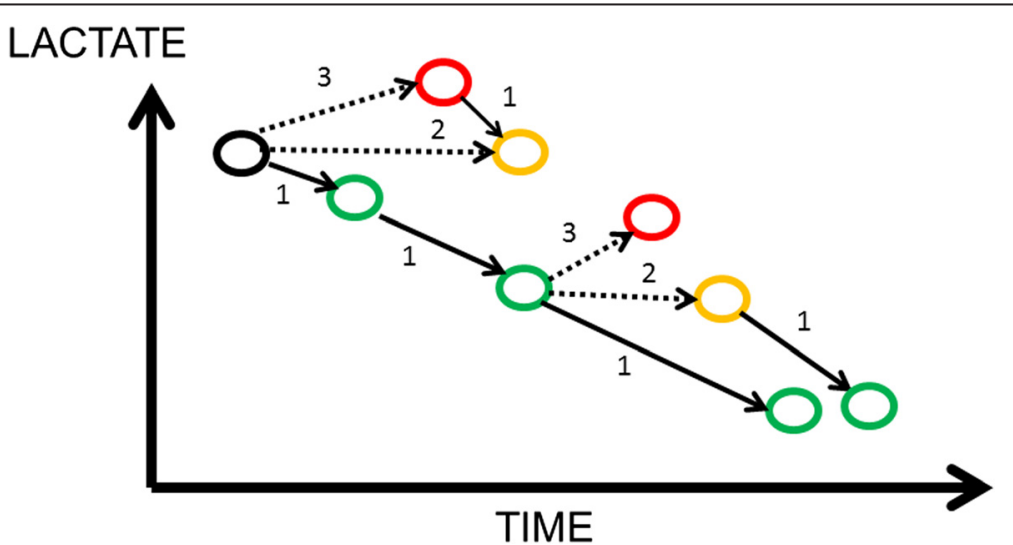

Fig. 1 Schematic showing some of the possible evolutions of blood lactate levels over time: decreasing (1), remaining stable (2), or increasing (3). Dashed lines represent an unfavorable course and suggest the need for treatment to be reviewed, if this has not already been done, because the current management is likely ineffective 


\section{Results}

A total of 96 studies met our inclusion criteria (Fig. 2, Table 1).

\section{General ICU patients}

\section{Observational studies}

We identified 13 observational studies in heterogeneous critically ill populations $[6,10-12,23-31]$. All of these studies indicated that nonsurvivors had persistently higher lactate concentrations over time than survivors. Only one study [26] reported that lactate reduction during the first 24 hrs of ICU stay was useful only in septic patients, but not in patients with hemorrhage or other conditions.
The suggested optimal timing of lactate measurements was not precisely defined in several of the studies that evaluated the course of lactate concentrations over time. The studies that did include a time interval usually selected 6,12 or even 24 hrs.

\section{Interventional studies}

An interventional trial of 348 patients by Jansen et al. [15] targeted a lactate decrease of at least $20 \%$ in 2 hrs for the initial $8 \mathrm{hrs}$ of treatment in ICU patients with an initial lactate $\geq 3 \mathrm{mEq} / \mathrm{l}$. This strategy was associated with a lower mortality rate in the lactate-guided therapy group after adjustment for predefined risk factors (hazard ratio (HR), 0.61; confidence interval (CI), 0.43-0.87).

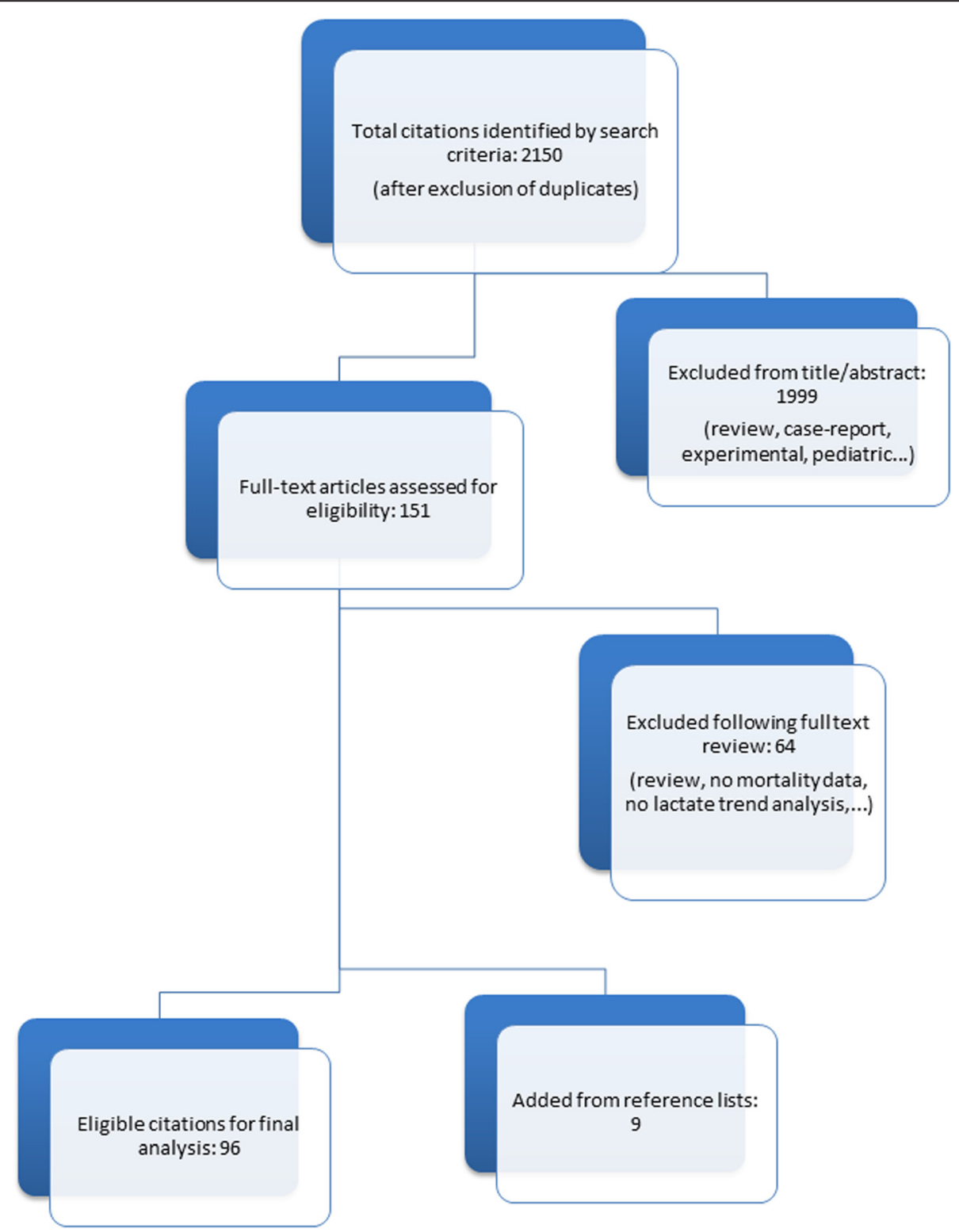

Fig. 2 Prisma diagram 
Table 1 Included studies according to population type

\begin{tabular}{|c|c|c|c|c|c|c|c|}
\hline & $\begin{array}{l}\text { First author, year } \\
\text { [reference] }\end{array}$ & $\begin{array}{l}\text { Number of } \\
\text { patients }\end{array}$ & Study design & $\begin{array}{l}\text { Initial minimum } \\
\text { lactate for } \\
\text { patient inclusion }\end{array}$ & $\begin{array}{l}\text { Timing of } \\
\text { measurements }\end{array}$ & $\begin{array}{l}\text { Suggested } \\
\text { time interval }\end{array}$ & Comments \\
\hline & \multicolumn{7}{|c|}{ General ICU/emergency department } \\
\hline & \multicolumn{7}{|l|}{ Observational } \\
\hline 1. & Vincent, 1983 [12] & 17 & Prospective & $\geq 4$ & $\begin{array}{l}\text { Every } 20 \text { min during } \\
\text { first } 2 \mathrm{~h} \text { of ICU } \\
\text { treatment }\end{array}$ & $1 \mathrm{~h}$ & $\begin{array}{l}\text { Decrease }>10 \% \text { associated } \\
\text { with survival }\end{array}$ \\
\hline 2. & Cowan, 1984 [23] & 30 & Prospective & - & $3 \mathrm{~h}, 24 \mathrm{~h}$ & $3 \mathrm{~h}$ & $\begin{array}{l}\text { Change in lactate predictive of } \\
\text { outcome but less so than } \\
\text { simple hemodynamic variables }\end{array}$ \\
\hline 3. & Suistomaa, 2000 [24] & 100 & Prospective & - & Every $2 \mathrm{~h}$ for $24 \mathrm{~h}$ & $6 \mathrm{~h}$ & $\begin{array}{l}\text { Failure to decrease lactate at } \\
6 \mathrm{~h} \text { associated with higher } \\
\text { mortality }\end{array}$ \\
\hline 4. & Jansen, 2008 [31] & 106 & Prospective & - & $\begin{array}{l}\text { Variable (at } \\
\text { ambulance pickup } \\
\text { and at ER arrival) }\end{array}$ & - & $\begin{array}{l}\text { Decrease in lactate } \\
\text { independently associated with } \\
\text { decreased hazard of death }\end{array}$ \\
\hline 5. & Wang, 2009 [25] & 101 & NR & $\geq 2$ & $12 \mathrm{~h}, 24 \mathrm{~h}$ & $12 \mathrm{~h}$ & $\begin{array}{l}\text { Decrease } \leq 10 \% \text { associated } \\
\text { with increased mortality }\end{array}$ \\
\hline 6. & Jansen, 2009 [26] & 394 & Prospective & - & $12 \mathrm{~h}, 24 \mathrm{~h}$ & $12 \mathrm{~h}$ & $\begin{array}{l}\text { Decrease in lactate only of } \\
\text { prognostic value in patients } \\
\text { with sepsis }\end{array}$ \\
\hline 7. & Krishna, 2009 [27] & 50 & Prospective & - & $12 \mathrm{~h}, 24 \mathrm{~h}, 36 \mathrm{~h}$ & $24 h, 36 h$ & $\begin{array}{l}\text { Decreasing levels associated } \\
\text { with survival }\end{array}$ \\
\hline 8. & Soliman, 2010 [28] & 433 & Prospective & - & $24 \mathrm{~h}, 48 \mathrm{~h}$ & $24 \mathrm{~h}$ & $\begin{array}{l}\text { Higher lactate concentrations } \\
\text { at } 24 \text { and } 48 \mathrm{~h} \text { after admission } \\
\text { associated with decreased } \\
\text { survival }\end{array}$ \\
\hline 9. & Nichol, 2010 [6] & 7155 & Retrospective & - & Variable & $24 \mathrm{~h}$ & $\begin{array}{l}\text { Time-weighted average lactate } \\
\text { over } 24 \mathrm{~h} \text { independent } \\
\text { predictor of mortality }\end{array}$ \\
\hline 10. & Nichol, 2011 [10] & 5041 & Retrospective & - & Variable & $24 \mathrm{~h}$ & $\begin{array}{l}\text { Time-weighted average lactate } \\
\text { and change in lactate over } \\
24 \mathrm{~h} \text { independent predictors of } \\
\text { hospital mortality }\end{array}$ \\
\hline 11. & van Beest, 2013 [29] & 2251 & Retrospective & - & Variable & $6 \mathrm{~h}$ & $\begin{array}{l}\text { Normalization of lactate }<6 \mathrm{~h} \\
\text { after ICU admission associated } \\
\text { with better hospital survival } \\
\text { than normalization of lactate } \\
>6 \text { hrs }\end{array}$ \\
\hline 12. & Zhang, 2014 [30] & 6291 & Retrospective & $>2$ & Variable & Variable & $\begin{array}{l}\text { Normalization and speed of } \\
\text { normalization related to } \\
\text { outcome }\end{array}$ \\
\hline \multirow[t]{2}{*}{13.} & Haas, 2016 [11] & 400 & Retrospective & $>10$ & Variable & $12 \mathrm{~h}$ & $\begin{array}{l}\text { No decrease in lactate over } \\
12 \mathrm{~h} \text { associated with increased } \\
\text { mortality }\end{array}$ \\
\hline & \multicolumn{7}{|l|}{ Interventional } \\
\hline 14. & Jansen, 2010 [15] & 348 & Prospective & $\geq 3.0$ & $2 \mathrm{~h}$ & $8 \mathrm{~h}$ & $\begin{array}{l}\text { Objective was to decrease } \\
\text { lactate by } 20 \% \text { or more per } \\
2 \mathrm{~h} \text { for the initial } 8 \mathrm{~h} \text { of ICU } \\
\text { stay. Lactate-guided therapy } \\
\text { was independently associated } \\
\text { with reduced hospital mortality }\end{array}$ \\
\hline & \multicolumn{7}{|l|}{ Surgical ICU } \\
\hline 15. & McNelis, 2001 [32] & 95 & Retrospective & - & $\begin{array}{l}\text { 8-h intervals until } \\
\text { lactate normalized }\end{array}$ & Variable & $\begin{array}{l}\text { Time to lactate normalization } \\
\text { predictive of outcome }\end{array}$ \\
\hline 16. & Husain, 2003 [33] & 137 & Retrospective & - & Variable & Variable & $\begin{array}{l}\text { Time to lactate normalization } \\
\text { independent predictor of } \\
\text { mortality }\end{array}$ \\
\hline
\end{tabular}


Table 1 Included studies according to population type (Continued)

\begin{tabular}{|c|c|c|c|c|c|c|c|}
\hline 17. & Meregalli, 2004 [34] & 44 & Prospective & - & 12 h, 24 h, 48 h & $48 \mathrm{~h}$ & $\begin{array}{l}\text { Blood lactate concentrations } \\
\text { decreased with time in } \\
\text { survivors, but remained stable } \\
\text { in nonsurvivors }\end{array}$ \\
\hline 18. & $\begin{array}{l}\text { Cardinal Fernandez, } \\
2009 \text { [35] }\end{array}$ & 108 & Prospective & $>2$ & $6 \mathrm{~h}$ & $6 \mathrm{~h}$ & $\begin{array}{l}\text { Decrease in lactate by }>40 \% \\
\text { associated with increased } \\
\text { survival }\end{array}$ \\
\hline \multirow[t]{2}{*}{19.} & Ibrahim, 2013 [36] & 322 & Prospective & - & $8 \mathrm{~h}, 16 \mathrm{~h}, 24 \mathrm{~h}$ & $16 \mathrm{~h}$ & $\begin{array}{l}\text { Percent change in blood } \\
\text { lactate at } 16 \mathrm{~h} \text { independent } \\
\text { predictor of postoperative } \\
\text { mortality }\end{array}$ \\
\hline & \multicolumn{7}{|l|}{ Cardiac surgery } \\
\hline 20. & Lindsay, 2013 [37] & 1291 & Retrospective & - & Variable & Variable & $\begin{array}{l}\text { Longer predicted time to reach } \\
\text { normal lactate }(<1.5 \mathrm{mmol} / \mathrm{l}) \\
\text { associated with increased } \\
\text { mortality }\end{array}$ \\
\hline 21. & Hajjar, 2013 [38] & 502 & Prospective & - & $6 \mathrm{~h}, 12 \mathrm{~h}$ & $6 \mathrm{~h}, 12 \mathrm{~h}$ & $\begin{array}{l}\text { Failure to decrease lactate } \\
\text { associated with major } \\
\text { complications, including death }\end{array}$ \\
\hline 22. & Park, 2014 [39] & 115 & Retrospective & - & $6 \mathrm{~h}, 12 \mathrm{~h}, 24 \mathrm{~h}$ & $\begin{array}{l}6 \mathrm{~h}, 12 \mathrm{~h}, \\
24 \mathrm{~h}\end{array}$ & $\begin{array}{l}\text { Lack of decrease in lactate } \\
\text { predictive of mortality }\end{array}$ \\
\hline 23. & $\begin{array}{l}\text { Lopez-Delgado, } \\
2015 \text { [40] }\end{array}$ & 2935 & Prospective & - & $6 \mathrm{~h}, 12 \mathrm{~h}, 24 \mathrm{~h}$ & $24 \mathrm{~h}$ & $\begin{array}{l}\text { Later peak in lactate associated } \\
\text { with higher hospital and } \\
\text { long-term mortality }\end{array}$ \\
\hline 24. & Li, 2015 [41] & 123 & Retrospective & - & $6 \mathrm{~h}, 12 \mathrm{~h}$ & $12 \mathrm{~h}$ & $\begin{array}{l}\text { Lactate decrease predictive of } \\
\text { in-hospital mortality in patients } \\
\text { receiving ECMO }\end{array}$ \\
\hline
\end{tabular}

Trauma

Observational

25. Abramson, 1993 [42]

Prospective

$8 h, 16 h, 24 h, 36 h, \quad 24 h$ $48 \mathrm{~h}$

Normalization of lactate by $24 \mathrm{~h}$ associated with $100 \%$ survival

26. Manikis, 1995 [43]

Retrospective

At least three times a Variable day

Duration of hyperlactatemia correlated with the development of organ failure but not with mortality

27. Holm, $2000[44]$

Prospective

12 h, 24 h, 48 h, 72 h Variable

28. Cerovic, 2003 [45]

Prospective

Twice daily during first 2 days and once daily during next 3 days

29. Kamolz, 2005 [46]

Prospective

Variable

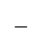

Retrospective

$$
\begin{aligned}
& \text { 2. Dubend } \\
& {[49]}
\end{aligned}
$$

33. Odom, 2013 [50]
623
Retrospective
$2 h, 4 h$

Variable

$6 \mathrm{~h}$
Decreasing lactate levels associated with survival

Reduced lactate levels in survivors

Higher mortality in patients with initial lactate $>2 \mathrm{mmol} / \mathrm{l}$ if lactate not normalized at $24 \mathrm{~h}$

Delayed or absent decrease in lactate associated with infectious complications but not mortality

Early normalization of lactate independent predictor of survival

In patients without traumatic brain injury, decrease in lactate impaired in nonsurvivors

Lower decrease in lactate at $6 \mathrm{~h}$ independently predictive of increased risk of death 
Table 1 Included studies according to population type (Continued)

\begin{tabular}{|c|c|c|c|c|c|c|c|}
\hline 34. & Heinonen, 2014 [51] & 610 & Retrospective & - & Variable & Variable & $\begin{array}{l}\text { Failure to normalize lactate } \\
\text { associated with increased } \\
\text { mortality }\end{array}$ \\
\hline 35. & Freitas, 2015 [52] & 117 & Retrospective & - & $6 \mathrm{~h}$ & $6 \mathrm{~h}$ & $\begin{array}{l}\text { No correlation between } \\
\text { decrease in lactate and } \\
\text { mortality }\end{array}$ \\
\hline \multirow[t]{2}{*}{36.} & Dezman, 2015 [53] & 3887 & Retrospective & $\geq 3$ & Variable & Variable & $\begin{array}{l}\text { No decrease in lactate } \\
\text { independent predictor of } \\
24-h \text { mortality }\end{array}$ \\
\hline & \multicolumn{7}{|l|}{ Interventional } \\
\hline 37. & Blow, 1999 [55] & 79 & Retrospective & - & Variable & $24 \mathrm{~h}$ & $\begin{array}{l}\text { Failure to decrease lactate } \\
\text { associated with increased } \\
\text { mortality }\end{array}$ \\
\hline \multirow[t]{3}{*}{38.} & Claridge, 2000 [56] & 364 & Prospective & - & Variable & $12 \mathrm{~h}$ & $\begin{array}{l}\text { Increase in infections, length of } \\
\text { stay, and mortality if lactate did } \\
\text { not normalize by } 12 \mathrm{~h}\end{array}$ \\
\hline & \multicolumn{7}{|l|}{ Sepsis } \\
\hline & \multicolumn{7}{|l|}{ Observational } \\
\hline 39. & Bakker, 1991 [57] & 48 & Prospective & $>2$ & Variable & Variable & $\begin{array}{l}\text { Only survivors had a significant } \\
\text { decrease in blood lactate } \\
\text { concentrations during the } \\
\text { course of septic shock }\end{array}$ \\
\hline 40. & Friedman, 1995 [58] & 35 & Prospective & $>2$ & $4 \mathrm{~h}, 24 \mathrm{~h}$ & Variable & $\begin{array}{l}\text { Lactate remained high in } \\
\text { nonsurvivors and progressively } \\
\text { decreased in survivors }\end{array}$ \\
\hline 41. & Bernardin, 1996 [59] & 32 & Prospective & - & $24 \mathrm{~h}$ & $24 \mathrm{~h}$ & $\begin{array}{l}\text { Greater decrease in lactate in } \\
\text { survivors }\end{array}$ \\
\hline 42. & Marecaux, 1996 [60] & 38 & Prospective & $>2$ & $24 h, 48 h$ & $24 \mathrm{~h}, 48 \mathrm{~h}$ & $\begin{array}{l}\text { Greater decrease in lactate in } \\
\text { survivors }\end{array}$ \\
\hline 43. & Bakker, 1996 [61] & 87 & Prospective & $>2$ & Variable & Variable & $\begin{array}{l}\text { Duration of lactic acidosis best } \\
\text { discriminant of survival }\end{array}$ \\
\hline 44. & Kobayashi, 2001 [62] & 22 & Prospective & - & $\begin{array}{l}\text { Every } 4 \text { hours for } \\
4 \text { days }\end{array}$ & Variable & $\begin{array}{l}\text { Decrease in lactate associated } \\
\text { with survival }\end{array}$ \\
\hline 45. & Nguyen, 2004 [63] & 111 & Prospective & - & $6 \mathrm{~h}$ & $6 \mathrm{~h}$ & $\begin{array}{l}\text { Decrease in lactate } \geq 10 \% \\
\text { associated with lower } 60 \text {-day } \\
\text { mortality }\end{array}$ \\
\hline 46. & Nguyen, 2007 [64] & 330 & Prospective & - & Variable & $6 \mathrm{~h}$ & $\begin{array}{l}\text { Decreased odds ratio for } \\
\text { mortality in patients with } \\
\text { decreased lactate }\end{array}$ \\
\hline 47. & Phua, 2008 [65] & 72 & Prospective & - & $24 \mathrm{~h}, 48 \mathrm{~h}$ & $24 \mathrm{~h}$ & $\begin{array}{l}\text { Increase in lactate predictive of } \\
\text { mortality }\end{array}$ \\
\hline 48. & Yang, 2009 [66] & 105 & Prospective & - & $6 \mathrm{~h}, 24 \mathrm{~h}, 72 \mathrm{~h}$ & $6 \mathrm{~h}$ & $\begin{array}{l}\text { Decrease in lactate at } 6 \mathrm{~h} \\
\geq 30 \% \text { was independent } \\
\text { predictor of survival }\end{array}$ \\
\hline 49. & Arnold, 2009 [67] & 166 & Retrospective & - & $6 \mathrm{~h}$ & $6 \mathrm{~h}$ & $\begin{array}{l}\text { Lactate decrease by less than } \\
10 \% \text { independent predictor of } \\
\text { in-hospital death }\end{array}$ \\
\hline 50. & Nguyen, 2010 [68] & 220 & Retrospective & - & $6 \mathrm{~h}$ & $6 \mathrm{~h}$ & $\begin{array}{l}\text { Larger decrease in lactate } \\
\text { associated with decreased } \\
\text { mortality up to } 12 \text { months }\end{array}$ \\
\hline 51. & Nguyen, 2011 [18] & 556 & Prospective & - & $12 \mathrm{~h}$ & $12 \mathrm{~h}$ & $\begin{array}{l}\text { Any decrease in lactate within } \\
12 \mathrm{~h} \text { from baseline or an initial } \\
\text { lactate }<2 \mathrm{mmol} / \mathrm{l} \\
\text { independently associated with } \\
\text { reduced mortality }\end{array}$ \\
\hline 52. & Puskarich, 2012 [69] & 203 & $\begin{array}{l}\text { Retrospective } \\
\text { analysis of data } \\
\text { from [16] }\end{array}$ & - & $2 \mathrm{~h}, 4 \mathrm{~h}, 6 \mathrm{~h}$ & $6 \mathrm{~h}$ & $\begin{array}{l}\geq 10 \% \text { decrease in lactate } \\
\text { during resuscitation associated } \\
\text { with decreased mortality }\end{array}$ \\
\hline
\end{tabular}


Table 1 Included studies according to population type (Continued)

\begin{tabular}{|c|c|c|c|c|c|c|c|}
\hline 53. & Zanaty, 2012 [70] & 53 & Prospective & - & $6 \mathrm{~h}$ & $6 \mathrm{~h}$ & $\begin{array}{l}<15 \% \text { decrease in lactate } \\
\text { independent predictor of } \\
\text { mortality }\end{array}$ \\
\hline 54. & Puskarich, 2013 [71] & 187 & $\begin{array}{l}\text { Retrospective } \\
\text { analysis of data } \\
\text { from [16] }\end{array}$ & - & $\begin{array}{l}\text { At least two lactate } \\
\text { measurements in first } \\
6 \mathrm{~h}\end{array}$ & $6 \mathrm{~h}$ & $\begin{array}{l}\text { Lactate normalization in } 6 \mathrm{~h} \\
\text { stronger independent predictor } \\
\text { of survival than decrease in } \\
\text { lactate by } \geq 50 \%\end{array}$ \\
\hline 55. & Walker, 2013 [72] & 78 & Retrospective & - & $6 \mathrm{~h}$ & $6 \mathrm{~h}$ & $\begin{array}{l}\text { Decrease in lactate } \\
\text { independently associated with } \\
\text { mortality, with optimal cut-off } \\
\text { of } 36 \%\end{array}$ \\
\hline 56. & Liu, 2013 [73] & 9190 & Retrospective & $\geq 2$ & $4 \mathrm{~h}, 8 \mathrm{~h}, 12 \mathrm{~h}$ & $12 \mathrm{~h}$ & $\begin{array}{l}\text { Reduced mortality in patients } \\
\text { with more than } 60 \% \text { lactate } \\
\text { improvement at } 12 \mathrm{~h} \text {. }\end{array}$ \\
\hline 57. & Marty, 2013 [74] & 94 & Prospective & - & $6 \mathrm{~h}, 12 \mathrm{~h}, 24 \mathrm{~h}$ & $24 \mathrm{~h}$ & $\begin{array}{l}\text { Decrease in lactate at } 24 \mathrm{~h} \\
\text { independently correlated to } \\
\text { survival }\end{array}$ \\
\hline 58. & Park, 2014 [75] & 25 & Prospective & - & $\begin{array}{l}6 \mathrm{~h}, 12 \mathrm{~h}, 18 \mathrm{~h}, 24 \mathrm{~h}, \\
48 \mathrm{~h}\end{array}$ & $48 \mathrm{~h}$ & $\begin{array}{l}\text { Normalization independent } \\
\text { predictor of survival }\end{array}$ \\
\hline 59. & Permpikul, 2014 [76] & 51 & Prospective & - & $6 \mathrm{~h}$ & $6 \mathrm{~h}$ & $\begin{array}{l}\text { Lactate decrease associated } \\
\text { with reduced 28-day mortality }\end{array}$ \\
\hline 60. & Bao, 2015 [77] & 94 & Retrospective & - & $3 \mathrm{~h}, 6 \mathrm{~h}, 24 \mathrm{~h}$ & $24 \mathrm{~h}$ & $\begin{array}{l}\text { 24-h lactate decrease predictive } \\
\text { of outcome }\end{array}$ \\
\hline 61. & Galbois, 2015 [78] & 42 & Prospective & - & 6 h, 12 h, 18 h, 24 h & $6 \mathrm{~h}$ & $\begin{array}{l}\text { Lesser decrease in lactate } \\
\text { associated with 14-day } \\
\text { mortality }\end{array}$ \\
\hline 62. & Lee, 2015 [79] & 109 & Retrospective & $>3.3$ & $6 h, 24 h, 48 h$ & $\begin{array}{l}6 \mathrm{~h}, 24 \mathrm{~h}, \\
48 \mathrm{~h}\end{array}$ & $\begin{array}{l}\text { Decrease in lactate of }<10 \% \text { in } \\
\text { the first } 6 \mathrm{~h}, 24 \mathrm{~h} \text {, and } 48 \mathrm{~h} \\
\text { independently associated with } \\
\text { mortality }\end{array}$ \\
\hline 63. & Dettmer, 2015 [17] & 243 & Retrospective & $\geq 4$ & Variable & Variable & $\begin{array}{l}\text { Greater reduction in lactate } \\
\text { associated with decreased } \\
\text { 28-day mortality }\end{array}$ \\
\hline 64. & $\begin{array}{l}\text { Lokhandwala, } 2015 \\
\text { [80] }\end{array}$ & 74 & Retrospective & $\geq 4$ & Variable & Variable & $\begin{array}{l}\text { Lactate decrease }<4 \mathrm{mmol} / / \mathrm{l} \\
\text { associated with increased } \\
\text { hospital morality }\end{array}$ \\
\hline 65. & Wang, 2015 [81] & 115 & Prospective & - & 6 h, 12 h, 18 h, 24 h & $24 \mathrm{~h}$ & $\begin{array}{l}\text { Lower lactate area score and } \\
\text { percentage decrease in lactate } \\
\text { associated with increased } \\
\text { mortality }\end{array}$ \\
\hline 66. & Bhat, 2015 [82] & 207 & Retrospective & - & Variable & Variable & $\begin{array}{l}\text { Higher mortality in patients } \\
\text { with no decrease in lactate }\end{array}$ \\
\hline 67. & Chertoff, 2016 [83] & 229 & Retrospective & - & $24-48 \mathrm{~h}$ & $24-48 \mathrm{~h}$ & $\begin{array}{l}\text { Lower decrease in plasma } \\
\text { lactate } 24-48 \mathrm{~h} \text { after initiation } \\
\text { of treatment was associated } \\
\text { with higher } 30 \text {-day mortality }\end{array}$ \\
\hline 68. & Drumheller, 2016 [84] & 411 & Retrospective & $\geq 4$ & Variable & Variable & $\begin{array}{l}\text { Decrease in lactate } \\
\text { independently associated with } \\
\text { decreased risk of death }\end{array}$ \\
\hline 69. & He, 2016 [85] & 84 & Prospective & - & $8 \mathrm{~h}$ & $8 \mathrm{~h}$ & $\begin{array}{l}\text { Patients with lactate decrease } \\
\geq 10 \% \text { had lower ICU mortality } \\
\text { than those with lactate } \\
\text { decrease }<10 \%\end{array}$ \\
\hline 70. & На, 2016 [86] & 208 & & - & $6 \mathrm{~h}, 24 \mathrm{~h}$ & $24 \mathrm{~h}$ & $\begin{array}{l}\text { Low decrease in lactate at } 6 \\
\text { and } 24 \mathrm{~h} \text { independently } \\
\text { associated with hospital } \\
\text { mortality, but } 24-\mathrm{h} \text { lactate } \\
\text { decrease had higher } \\
\text { discriminatory power }\end{array}$ \\
\hline
\end{tabular}


Table 1 Included studies according to population type (Continued)

\begin{tabular}{|c|c|c|c|c|c|c|c|}
\hline 71. & Bolvardi, 2016 [87] & 90 & Prospective & - & $6 \mathrm{~h}$ & $6 \mathrm{~h}$ & $\begin{array}{l}\text { Lactate decrease }<10 \% \\
\text { associated with increased } \\
\text { mortality }\end{array}$ \\
\hline \multirow[t]{2}{*}{72.} & Amir, 2016 [88] & 202 & Prospective & - & $6 \mathrm{~h}$ & $6 \mathrm{~h}$ & $\begin{array}{l}\text { Lactate decrease } \geq 10 \% \text { not } \\
\text { associated with mortality }\end{array}$ \\
\hline & Interventional & & & & & & \\
\hline 73. & Jones, 2010 [16] & 300 & Prospective & - & Variable & Variable & $\begin{array}{l}\text { No differences in in-hospital } \\
\text { mortality using management } \\
\text { to normalize lactate compared } \\
\text { with management to normalize } \\
\mathrm{ScvO}_{2}\end{array}$ \\
\hline 74. & Tian, 2012 [89] & 62 & Prospective & - & Variable & $48 \mathrm{~h}$ & $\begin{array}{l}\text { 28-day mortality rates lower in } \\
\text { patients with } 30 \% \text { decrease in } \\
\text { lactate target than in those } \\
\text { with } 10 \% \text { decrease in lactate } \\
\text { target and controls }\end{array}$ \\
\hline 75. & Yu, 2013 [90] & 50 & Prospective & - & $3 \mathrm{~h}, 6 \mathrm{~h}, 72 \mathrm{~h}$ & $6 \mathrm{~h}, 72 \mathrm{~h}$ & $\begin{array}{l}\text { No differences in in-hospital } \\
\text { mortality using management } \\
\text { targeted at } 10 \% \text { lactate decrease } \\
\text { compared with management to } \\
\text { normalize } \mathrm{ScVO}_{2}\end{array}$ \\
\hline 76. & Lyu, 2015 [91] & 100 & Prospective & - & $\begin{array}{l}1 \mathrm{~h}, 2 \mathrm{~h}, 3 \mathrm{~h}, 4 \mathrm{~h}, 5 \mathrm{~h}, \\
6 \mathrm{~h}\end{array}$ & $6 \mathrm{~h}$ & $\begin{array}{l}\text { 28-day mortality independently } \\
\text { associated with lactate } \\
\text { decrease }<10 \%\end{array}$ \\
\hline \multirow[t]{2}{*}{77.} & Kuan, 2016 [92] & 122 & Prospective & $\geq 3$ & Variable & $3 \mathrm{~h}$ & $\begin{array}{l}\text { Lactate decrease }>20 \% \\
\text { associated with decreased } \\
\text { mortality }\end{array}$ \\
\hline & \multicolumn{7}{|l|}{ Cardiogenic shock } \\
\hline 78. & Attana, 2012 [93] & 51 & Prospective & - & $12 \mathrm{~h}$ & $12 \mathrm{~h}$ & $\begin{array}{l}\text { Decrease in lactate by }<10 \% \\
\text { predicts higher risk of death }\end{array}$ \\
\hline 79. & Attana, 2013 [94] & 63 & Prospective & - & $12 \mathrm{~h}$ & $12 \mathrm{~h}$ & $\begin{array}{l}\text { Nonsurvivors had smaller } \\
\text { decrease in lactate }\end{array}$ \\
\hline 80. & Park, 2014 [95] & 96 & Retrospective & - & Variable & $48 \mathrm{~h}$ & $\begin{array}{l}\text { Lactate decrease }<70 \% \\
\text { independent predictor of } \\
\text { hospital mortality }\end{array}$ \\
\hline \multirow[t]{2}{*}{81.} & Guenther, 2014 [96] & 41 & retrospective & - & Variable & $6 \mathrm{~h}$ & $\begin{array}{l}\text { Increased lactate } \\
\text { concentrations at } 6 \mathrm{~h} \\
\text { associated with nonsurvival } \\
\text { after ECMO }\end{array}$ \\
\hline & Cardiac arrest & & & & & & \\
\hline 82. & Kliegel, 2004 [97] & 394 & Retrospective & - & $24 h, 48 h$ & $48 \mathrm{~h}$ & $\begin{array}{l}\text { Persistent hyperlactatemia } \\
\text { predictive or poor prognosis }\end{array}$ \\
\hline 83. & Donnino, 2007 [98] & 79 & Retrospective & - & $6 \mathrm{~h}, 12 \mathrm{~h}$ & $12 \mathrm{~h}$ & $\begin{array}{l}\text { Decrease in lactate } \\
\text { independent predictor of } \\
\text { hospital survival }\end{array}$ \\
\hline 84. & Arnalich, 2010 [99] & 85 & Prospective & - & $6 \mathrm{~h}$ & $6 \mathrm{~h}$ & $\begin{array}{l}\text { Decrease in lactate significantly } \\
\text { higher in 24-h survivors com- } \\
\text { pared with nonsurvivors }\end{array}$ \\
\hline 85. & Le Guen, 2011 [100] & 51 & Prospective & - & $1 \mathrm{~h}$ & $1 \mathrm{~h}$ & $\begin{array}{l}\text { Decrease in blood lactate } \\
>10 \% \text { significantly different in } \\
\text { survivors and nonsurvivors } \\
\text { treated with ECMO }\end{array}$ \\
\hline 86. & Starodub, 2013 [101] & 199 & Retrospective & - & $6 \mathrm{~h}, 12 \mathrm{~h}, 24 \mathrm{~h}$ & $12 \mathrm{~h}, 24 \mathrm{~h}$ & $\begin{array}{l}\text { Change in lactate over time } \\
\text { not predictive of survival but } \\
\text { lower mean lactate levels at } 12 \\
\text { and } 24 \mathrm{~h} \text { associated with } \\
\text { increased survival }\end{array}$ \\
\hline
\end{tabular}


Table 1 Included studies according to population type (Continued)

\begin{tabular}{|c|c|c|c|c|c|c|c|}
\hline 87. & Donnino, 2014 [102] & 100 & Prospective & $\overline{-}$ & $12 \mathrm{~h}, 24 \mathrm{~h}$ & $12 \mathrm{~h}$ & $\begin{array}{l}\text { Greater percentage decrease } \\
\text { independently associated with } \\
\text { survival }\end{array}$ \\
\hline 88. & Riveiro, 2015 [103] & 54 & Prospective & - & $\begin{array}{l}6 h, 12 h, 24 h, \\
48 h, 72 h\end{array}$ & $6 \mathrm{~h}$ & $\begin{array}{l}\text { Decrease in lactate predictive } \\
\text { of 28-day survival }\end{array}$ \\
\hline \multirow[t]{2}{*}{89.} & Williams, 2016 [104] & 167 & Retrospective & - & Variable & $4 \mathrm{~h}$ & $\begin{array}{l}\text { More rapid decrease in lactate } \\
\text { in survivors }\end{array}$ \\
\hline & \multicolumn{7}{|l|}{ Respiratory failure } \\
\hline 90. & Zhao, 2010 [105] & 110 & Prospective & - & $6 \mathrm{~h}$ & $6 \mathrm{~h}$ & $\begin{array}{l}\text { Lactate decrease } \geq 10 \% \\
\text { associated with improved } \\
\text { survival }\end{array}$ \\
\hline 91. & Wu, 2012 [106] & 27 & Prospective & - & 12 h, 24 h, 48 h, 72 h & $\begin{array}{l}12 \mathrm{~h}, 24 \mathrm{~h}, \\
48 \mathrm{~h}, 72 \mathrm{~h}\end{array}$ & $\begin{array}{l}\text { Smaller decrease in lactate } \\
\text { predictive of outcome }\end{array}$ \\
\hline \multirow[t]{2}{*}{92.} & Zang, 2014 [107] & 43 & Prospective & - & $6 \mathrm{~h}$ & $6 \mathrm{~h}$ & $\begin{array}{l}\text { Decrease in lactate } \\
\text { independent predictor of } \\
\text { survival in patients treated } \\
\text { by ECMO }\end{array}$ \\
\hline & \multicolumn{7}{|l|}{ Others } \\
\hline 93. & Scott, 2010 [110] & 95 & Prospective & - & $1 \mathrm{~h}, 2 \mathrm{~h}, 6 \mathrm{~h}, 24 \mathrm{~h}$ & $2 \mathrm{~h}$ & $\begin{array}{l}\text { Lactate decrease }<15 \% \\
\text { predictive of poor outcome } \\
\text { (hospital mortality or } \\
\text { endotracheal intubation) in } \\
\text { patients with cardiorespiratory } \\
\text { insufficiency }\end{array}$ \\
\hline 94. & Wu, 2011 [109] & 222 & Prospective & - & $6 \mathrm{~h}$ & $6 \mathrm{~h}$ & $\begin{array}{l}\text { Lactate decrease of }<24.8 \% \text { at } \\
6 \mathrm{~h} \text { associated with higher } \\
\text { incidence of liver graft failure } \\
\text { and mortality }\end{array}$ \\
\hline 95. & Lui, 2013 [108] & 204 & Prospective & $\geq 2$ & $12 \mathrm{~h}$ & $12 \mathrm{~h}$ & $\begin{array}{l}\text { Smaller decrease in lactate } \\
\text { associated with increased } \\
\text { mortality in patients with } \\
\text { paraquat poisoning }\end{array}$ \\
\hline 96. & Mohamed, 2014 [111] & 46 & Prospective & - & $8 \mathrm{~h}, 24 \mathrm{~h}$ & $24 \mathrm{~h}$ & $\begin{array}{l}\text { Mortality greater if }<40 \% \\
\text { decrease in lactate }\end{array}$ \\
\hline
\end{tabular}

$\overline{E M C O}$ extracorporeal membrane oxygenation, $E R$ emergency room, $h$ hours, $N R$ not reported, $\mathrm{ScvO}_{2}$ central venous oxygen saturation

\section{Surgical patients}

We identified five observational studies conducted in general surgical ICU patients [32-36]. Failure of lactate concentrations to decrease over time was associated with worse outcomes in all studies.

\section{After cardiac surgery}

There were five observational studies in cardiac surgery patients [37-41], including two studies in patients treated with extracorporeal membrane oxygenation (ECMO) post cardiac surgery $[39,41]$. All studies consistently demonstrated differences in changes in lactate concentration between survivors and nonsurvivors.

\section{Trauma patients}

\section{Observational studies}

We identified twelve observational studies in trauma patients [42-53]. Three retrospective studies reported no association of change in lactate levels with mortality [43, 47, 52], although Manikis et al. [43] reported that the duration of hyperlactatemia was associated with the development of organ failure and Billeter et al. [47] noted that delayed or no reduction in blood lactate was associated with increased infectious complications. Several small studies used relatively long time intervals of 12-24 hrs $[45,54]$. One study reported that repeated lactate after 2 hrs could be valuable [48] and a retrospective study proposed a time limit of $6 \mathrm{hrs}$ [50].

\section{Interventional studies}

In a retrospective analysis of a small prospective cohort managed according to a protocol to normalize blood lactate levels, Blow et al. [55] reported that failure to normalize blood lactate levels $(<2.5 \mathrm{mmol} / \mathrm{l})$ was associated with increased morbidity and mortality. In an interventional study by Claridge et al. [56], patients were managed according to the same protocol targeted at reducing lactate levels to $<2.4 \mathrm{mmol} / \mathrm{l}$. Failure to achieve this target was associated with increased risk of infection, increased length of stay, and increased mortality. 


\section{Patients with sepsis Observational studies}

We found thirty four observational studies in patients with sepsis [17, 18, 57-88]. One study reported that a decrease in lactate levels of $\geq 10 \%$ was not associated with mortality [88], but this study was conducted in a low-resource setting, such that resuscitation may not have been optimal as acknowledged by the authors. Several studies reported that 6-hrly changes could be a useful guide [63, 64, 66, 67, 69-72, 78].

\section{Interventional studies}

One interventional study by Jones et al. [16] compared resuscitation based on lactate concentrations with a target of obtaining a $>10 \%$ decrease from the initial value with resuscitation based on achieving central venous oxygen saturation $\left(\mathrm{ScvO}_{2}\right) \geq 70 \%$; there were no differences in outcome between the two strategies. In an analysis of patients in this trial who had simultaneous lactate and $\mathrm{ScvO}_{2}$ measurements, Puskarich et al. [69] concluded that failure to achieve the target lactate decrease was associated with a worse prognosis than failure to achieve the $\mathrm{ScvO}_{2}$ target. In a small Chinese study [89], patients randomized to a $30 \%$ decrease in lactate target had better 28-day survival than those randomized to a $10 \%$ target or to control, and in another small study [90] there were no differences in in-hospital mortality using management targeted at a $10 \%$ decrease in lactate compared with management to normalize $\mathrm{ScvO}_{2}$. Two other Chinese studies reported that patients randomized to lactate-directed therapy had improved outcomes [91, 92].

\section{Patients with cardiogenic shock}

There were four studies in patients with cardiogenic shock [93-96], all showing that lactate concentrations decreased more in survivors than in nonsurvivors.

\section{After cardiac arrest}

We identified eight observational studies [97-104] in post-cardiac arrest patients. All but one [101] of these studies demonstrated differences in changes in lactate concentration between survivors and nonsurvivors.

\section{Patients with acute respiratory failure}

We found three observational studies in patients with acute respiratory failure [105-107], all showing that decreasing lactate levels were predictive of survival.

\section{Other conditions}

Changes in lactate concentrations were also reported following paraquat poisoning [108], after liver transplantation [109], in patients with acute cardiorespiratory failure [110], and in patients with severe community- acquired pneumonia [111]. All studies indicated the value of repeated lactate concentrations in these patient populations.

\section{Discussion}

Our literature review clearly supports the value of serial lactate measurements in the evaluation of critically ill patients and their response to therapy. This observation was similar across all studies and in all categories of patients, without being restricted to those with sepsis. We found only one study which suggested that evaluating the time course of lactate concentrations would be useful in sepsis patients but not in other conditions [26], and just five studies reporting no predictive effect of decrease in lactate levels over time on mortality $[43,47,52,88,101]$ although two of these did suggest a relationship with morbidity outcomes [43, 47]. Repeated lactate concentrations can also help separate patients with complications, such as neurological complications after cardiac arrest [112, 113] or after surgery [38]. A meta-analysis of these data is complicated by the heterogeneity of the populations and the different timings of the measurements, but the data are very consistent across studies.

Increased lactate concentrations can be due to factors other than cellular hypoxia, so the decrease in blood lactate concentrations may not just be the result of improvements in cellular oxygen availability. For example, beta-adrenergic stimulation may contribute to increased lactate production [114]. A recent study indicated the reverse phenomenon; that is, the increase in lactate concentrations seen in patients with sepsis may be blunted in patients previously treated with betablocking agents [115]. The infusion of lactate-containing intravenous solutions may also potentially complicate the interpretation of blood lactate concentrations [116], although the amount of fluid infused must be very large to have such an effect [117]. A recent study also reported that lactate levels decrease more slowly in patients with a positive blood alcohol level, thus complicating evaluation of blood lactate levels in these patients [118].

Because lactate is primarily metabolized in the liver, liver dysfunction may alter lactate clearance. Thus, some studies have questioned whether blood lactate concentrations can be used to indicate tissue hypoperfusion in critically ill patients with hepatic dysfunction. However, patients with stable cirrhosis have normal lactate concentrations [8]. Kruse et al. [119] analyzed the incidence of hyperlactatemia in patients with liver disease and showed that lactic acidosis was associated with clinical evidence of shock and increased hospital mortality. Chiolero et al. [120] reported that major hepatectomy was not associated 
with any global changes in lactate clearance, although lactate half-life was prolonged. A recent experimental study indicated that liver hypoperfusion is unlikely to contribute to increased blood lactate concentrations [121]. In patients with paracetamolinduced acute liver failure, higher lactate concentrations were associated with more severe organ failure and mortality [122].

Some investigators have compared lactate and $\mathrm{ScvO}_{2}$ or combined the two measures. Lactate is usually a better prognostic marker [69]. But is it actually necessary to choose? In an interventional study in patients with sepsis, Jones et al. [16] reported no differences in outcomes for patients managed according to lactate concentrations or to $\mathrm{ScvO}_{2}$ values, but it is difficult to evaluate how these measurements really guided therapy because there were no differences in administered treatments during the first $72 \mathrm{hrs}$. In post-cardiac surgery patients, Polonen et al. [14] reported better outcomes when $\mathrm{ScvO}_{2}$ and lactate concentrations were targeted together than in control patients. The most convincing evidence in favor of lactate as a target comes from the study by Jansen et al. [15] in which outcomes were improved in patients treated to a target of a $20 \%$ decrease in lactate concentrations. Nevertheless, the relatively slow changes in lactate make it difficult to interpret these results-the trend analysis is more a marker of effective treatment than a target in itself.

Although changes in blood lactate kinetics were clearly significant after $6 \mathrm{hrs}$ in many studies and after $12 \mathrm{hrs}$ in most, it is currently not possible to define the best time interval between lactate measurements. The normal reduction in lactate concentrations when overproduction of lactate abruptly ceases after grand mal seizures is about $50 \%$ in $1 \mathrm{hr}$ [13]. Although Levraut et al. [21] suggested that lactate clearance may be decreased in septic patients, Revelly et al. [22] reported similar values in patients with sepsis and in healthy volunteers.

The rate of lactate decrease in optimal treatment conditions is quite variable. In the best conditions, blood lactate concentrations decreased by more than $10 \%$ in $1 \mathrm{hr}$ in patients who responded rapidly to resuscitation [12] or by $10-20 \%$ in 2 hrs [15]. A study by Hernandez et al. [123] suggested a $>50 \%$ decrease in lactate concentrations during the first $6 \mathrm{hrs}$ of resuscitation in patients with septic shock. Although some systems now allow the quasi-continuous measurement of lactate concentrations, determinations every 1-2 hrs are probably sufficient; in the interventional study by Jansen et al. [15] the protocol was to measure blood lactate every 2 hrs. Even though serial blood lactate concentrations have been suggested to guide therapy, our review underlines that changes in lactate over time are relatively slow, taking place over hrs, and this may be too slow to guide therapy. Serial lactate concentrations should serve as a regular control, similar to how in the past a navigator would consult a compass from time to time to ensure that their boat was still heading in the right direction. If lactate concentrations do not normalize over time, the need for changes in therapy should be considered.

\section{Conclusion}

Our systematic literature review has provided the following answers to our initial questions. First, observation of a better prognosis with decreasing lactate concentrations is consistent throughout the literature. Second, these observations are not specific to septic patients, but apply to all common situations of hyperlactatemia and in heterogeneous patient populations. Third, the changes are relatively slow, and it is difficult to provide recommendations about the speed of decrease in lactate concentrations in the best conditions. Clearly repeating measurements every $12 \mathrm{hrs}$ can generally separate those who will do well from those who are likely to die, but shorter time intervals may be helpful. On the basis of our observations, we would recommend checking blood lactate concentrations as often as every 1-2 hrs in acute conditions. Fourth, the study of lactate kinetics appears to be valid regardless of the initial value and not only in patients with severe hyperlactatemia.

\section{Abbreviations \\ $\mathrm{ECMO}$, extracorporeal membrane oxygenation; $\mathrm{ScvO}_{2}$, central venous oxygen saturation \\ Acknowledgements \\ None. \\ Funding \\ Institutional funds only. \\ Availability of supporting data \\ Not applicable.

\begin{abstract}
Authors' contributions
AQeS and LC performed the literature search and drafted the manuscript. J-LV and FST reviewed the article for critical content. All authors read and approved the final manuscript.
\end{abstract}

Competing interests

The authors declare that they have no competing interests.

Consent for publication

Not applicable.

Ethics approval and consent to participate

Not applicable.

Received: 12 May 2016 Accepted: 1 July 2016

Published online: 13 August 2016 


\section{References}

1. Broder G, Weil MH. Excess lactate: an index of reversibility of shock in human patients. Science. 1964;143:1457-9.

2. Weil MH, Afifi AA. Experimental and clinical studies on lactate and pyruvate as indicators of the severity of acute circulatory failure (shock). Circulation. 1970:41:989-1001.

3. Peretz DI, McGregor M, Dossetor JB. Lactic acidosis: a clinically significant aspect of shock. Can Med Assoc J. 1964;90:673-5.

4. Vincent JL, De Backer D. Circulatory shock. N Engl J Med. 2013;369:1726-34.

5. Levy B. Lactate and shock state: the metabolic view. Curr Opin Crit Care. 2006;12:315-21.

6. Nichol AD, Egi M, Pettila V, Bellomo R, French C, Hart G, et al. Relative hyperlactatemia and hospital mortality in critically ill patients: a retrospective multi-centre study. Crit Care. 2010;14:R25.

7. Singer M, Deutschman CS, Seymour CW, Shankar-Hari M, Annane D, Bauer M, et al. The Third International Consensus Definitions for Sepsis and Septic Shock (Sepsis-3). JAMA. 2016:315:801-10.

8. Kraut JA, Madias NE. Lactic acidosis. N Engl J Med. 2014;371:2309-19.

9. Cecconi M, De Backer D, Antonelli M, Beale R, Bakker J, Hofer C, et al. Consensus on circulatory shock and hemodynamic monitoring. Task force of the European Society of Intensive Care Medicine. Intensive Care Med. 2014;40:1795-815

10. Nichol A, Bailey M, Egi M, Pettila V, French C, Stachowski E, et al. Dynamic lactate indices as predictors of outcome in critically ill patients. Crit Care. 2011;15:R242.

11. Haas SA, Lange T, Saugel B, Petzoldt M, Fuhrmann V, Metschke M, et al. Severe hyperlactatemia, lactate clearance and mortality in unselected critically ill patients. Intensive Care Med. 2016;42:202-10.

12. Vincent JL, Dufaye P, Berre J, Leeman M, Degaute JP, Kahn RJ. Serial lactate determinations during circulatory shock. Crit Care Med. 1983;1 1:449-51.

13. Orringer CE, Eustace JC, Wunsch CD, Gardner LB. Natural history of lactic acidosis after grand-mal seizures. A model for the study of an anion-gap acidosis not associated with hyperkalemia. N Engl J Med. 1977;297:796-9.

14. Polonen P, Ruokonen E, Hippelainen M, Poyhonen M, Takala J. A prospective, randomized study of goal-oriented hemodynamic therapy in cardiac surgical patients. Anesth Analg. 2000;90:1052-9.

15. Jansen TC, van Bommel J, Schoonderbeek FJ, Sleeswijk Visser SJ, van der Klooster JM, Lima AP, et al. Early lactate-guided therapy in intensive care unit patients: a multicenter, open-label, randomized controlled trial. Am J Respir Crit Care Med. 2010:182:752-61.

16. Jones AE, Shapiro NI, Trzeciak S, Arnold RC, Claremont HA, Kline JA. Lactate clearance vs central venous oxygen saturation as goals of early sepsis therapy: a randomized clinical trial. JAMA. 2010;303:739-46.

17. Dettmer M, Holthaus CV, Fuller BM. The impact of serial lactate monitoring on emergency department resuscitation interventions and clinical outcomes in severe sepsis and septic shock: an observational cohort study. Shock. 2015;43:55-61.

18. Nguyen HB, Kuan WS, Batech M, Shrikhande P, Mahadevan M, Li CH, et al. Outcome effectiveness of the severe sepsis resuscitation bundle with addition of lactate clearance as a bundle item: a multi-national evaluation. Crit Care. 2011;15:R229.

19. Vincent JL. Serial blood lactate levels reflect both lactate production and clearance. Crit Care Med. 2015;43:e209.

20. Vincent JL. Lactic acidosis. N Engl J Med. 2015;372:1077-8.

21. Levraut J, Ciebiera JP, Chave S, Rabary O, Jambou P, Carles M, et al. Mild hyperlactatemia in stable septic patients is due to impaired lactate clearance rather than overproduction. Am J Respir Crit Care Med. 1998;157:1021-6.

22. Revelly JP, Tappy L, Martinez A, Bollmann M, Cayeux MC, Berger MM, et al. Lactate and glucose metabolism in severe sepsis and cardiogenic shock. Crit Care Med. 2005:33:2235-40.

23. Cowan BN, Burns HJ, Boyle P, Ledingham IM. The relative prognostic value of lactate and haemodynamic measurements in early shock. Anaesthesia. 1984:39:750-5

24. Suistomaa M, Ruokonen E, Kari A, Takala J. Time-pattern of lactate and lactate to pyruvate ratio in the first 24 hrs of intensive care emergency admissions. Shock. 2000;14:8-12.

25. Wang H, Wu DW, Chen XM, Li C, Ding SF, Zhai Q, et al. Relationship between blood lactic level, lactic clearance, duration of lacticemia and prognosis of critically ill patients in intensive care unit. Zhongguo Wei Zhong Bing Ji Jiu Yi Xue. 2009;21:357-60.
26. Jansen TC, van Bommel J, Mulder PG, Lima AP, van der Hoven B, Rommes JH, et al. Prognostic value of blood lactate levels: does the clinical diagnosis at admission matter? J Trauma. 2009;66:377-85,

27. Krishna U, Joshi SP, Modh M. An evaluation of serial blood lactate measurement as an early predictor of shock and its outcome in patients of trauma or sepsis. Indian J Crit Care Med. 2009;13:66-73.

28. Soliman HM, Vincent JL. Prognostic value of admission serum lactate concentrations in intensive care unit patients. Acta Clin Belg. 2010;65:176-81.

29. van Beest PA, Brander L, Jansen SP, Rommes JH, Kuiper MA, Spronk PE. Cumulative lactate and hospital mortality in ICU patients. Ann Intensive Care. 2013;3:6.

30. Zhang Z, Chen $\mathrm{K}, \mathrm{Ni} \mathrm{H}$, Fan H. Predictive value of lactate in unselected critically ill patients: an analysis using fractional polynomials. J Thorac Dis. 2014:6:995-1003.

31. Jansen TC, van Bommel J, Mulder PG, Rommes JH, Schieveld SJ, Bakker J. The prognostic value of blood lactate levels relative to that of vital signs in the pre-hospital setting: a pilot study. Crit Care. 2008;12:R160.

32. McNelis J, Marini CP, Jurkiewicz A, Szomstein S, Simms HH, Ritter G, et al. Prolonged lactate clearance is associated with increased mortality in the surgical intensive care unit. Am J Surg. 2001:182:481-5.

33. Husain FA, Martin MJ, Mullenix PS, Steele SR, Elliott DC. Serum lactate and base deficit as predictors of mortality and morbidity. Am J Surg. 2003;185:485-91.

34. Meregalli A, Oliveira RP, Friedman G. Occult hypoperfusion is associated with increased mortality in hemodynamically stable, high-risk, surgical patients. Crit Care. 2004;8:R60-5.

35. Cardinal Fernandez PA, Olano E, Acosta C, Bertullo H, Albornoz H, Bagnulo $\mathrm{H}$. Prognostic value of lactate clearance in the first 6 hrs of intensive medicine course. Med Intensiva. 2009:33:166-70.

36. Ibrahim WA, Ahmed AS. Serial estimations of blood lactate predict postoperative outcome in cancer patients undergoing head and neck surgeries. Egyptian J Anaesth. 2013;29:149-54

37. Lindsay AJ, Xu M, Sessler DI, Blackstone EH, Bashr CA. Lactate clearance time and concentration linked to morbidity and death in cardiac surgical patients. Ann Thorac Surg. 2013;95:486-92.

38. Hajjar LA, Almeida JP, Fukushima JT, Rhodes A, Vincent JL, Osawa EA, et al. High lactate levels are predictors of major complications after cardiac surgery. J Thorac Cardiovasc Surg. 2013;146:455-60.

39. Park SJ, Kim SP, Kim JB, Jung SH, Choo SJ, Chung CH, et al. Blood lactate level during extracorporeal life support as a surrogate marker for survival. J Thorac Cardiovasc Surg. 2014;148:714-20.

40. Lopez-Delgado JC, Esteve F, Javierre C, Torrado H, Rodriguez-Castro D, Carrio ML, et al. Evaluation of serial arterial lactate levels as a predictor of hospital and long-term mortality in patients after cardiac surgery. J Cardiothorac Vasc Anesth. 2015;29:1441-53.

41. Li CL, Wang H, Jia M, Ma N, Meng X, Hou XT. The early dynamic behavior of lactate is linked to mortality in postcardiotomy patients with extracorporeal membrane oxygenation support: A retrospective observational study. J Thorac Cardiovasc Surg. 2015;149:1445-50.

42. Abramson D, Scalea TM, Hitchcock R, Trooskin SZ, Henry SM, Greenspan J. Lactate clearance and survival following injury. J Trauma. 1993;35:584-8.

43. Manikis P, Jankowski S, Zhang H, Kahn RJ, Vincent JL. Correlation of serial blood lactate levels to organ failure and mortality after trauma. Am J Emerg Med. 1995;13:619-22.

44. Holm C, Melcer B, Horbrand F, Worl HH, von Donnersmarck GH, Muhlbauer W. Haemodynamic and oxygen transport responses in survivors and non-survivors following thermal injury. Burns. 2000;26:25-33.

45. Cerovic O, Golubovic V, Spec-Marn A, Kremzar B, Vidmar G. Relationship between injury severity and lactate levels in severely injured patients. Intensive Care Med. 2003:29:1300-5.

46. Kamolz LP, Andel H, Schramm W, Meissl G, Herndon DN, Frey M. Lactate: early predictor of morbidity and mortality in patients with severe burns. Burns. 2005:31:986-90.

47. Billeter A, Turina M, Seifert B, Mica L, Stocker R, Keel M. Early serum procalcitonin, interleukin-6, and 24-hr lactate clearance: useful indicators of septic infections in severely traumatized patients. World J Surg. 2009:33:558-66

48. Regnier MA, Raux M, Le MY, Asencio Y, Gaillard J, Devilliers C, et al. Prognostic significance of blood lactate and lactate clearance in trauma patients. Anesthesiology. 2012;117:1276-88. 
49. Dubendorfer C, Billeter AT, Seifert B, Keel M, Turina M. Serial lactate and admission SOFA scores in trauma: an analysis of predictive value in 724 patients with and without traumatic brain injury. Eur J Trauma Emerg Surg 2013;39:25-34

50. Odom SR, Howell MD, Silva GS, Nielsen VM, Gupta A, Shapiro NI, et al. Lactate clearance as a predictor of mortality in trauma patients. J Trauma Acute Care Surg. 2013;74:999-1004.

51. Heinonen E, Hardcastle TC, Barle H, Muckart DJ. Lactate clearance predicts outcome after major trauma. Afr J Emerg Med. 2014;4:61-5.

52. Freitas $A D$, Franzon O. Lactate as predictor of mortality in polytrauma. Arq Bras Cir Dig. 2015;28:163-6.

53. Dezman ZD, Comer AC, Smith GS, Narayan M, Scalea TM, Hirshon JM. Failure to clear elevated lactate predicts $24-\mathrm{hr}$ mortality in trauma patients. J Trauma Acute Care Surg. 2015;79:580-5.

54. Roumen RM, Redl H, Schlag G, Sandtner W, Koller W, Goris RJ. Scoring systems and blood lactate concentrations in relation to the development of adult respiratory distress syndrome and multiple organ failure in severely traumatized patients. J Trauma. 1993;35:349-55.

55. Blow O, Magliore L, Claridge JA, Butler K, Young JS. The golden hr and the silver day: detection and correction of occult hypoperfusion within $24 \mathrm{hrs}$ improves outcome from major trauma. J Trauma. 1999;47:964-9.

56. Claridge JA, Crabtree TD, Pelletier SJ, Butler K, Sawyer RG, Young JS. Persistent occult hypoperfusion is associated with a significant increase in infection rate and mortality in major trauma patients. J Trauma. 2000;48:8-14

57. Bakker J, Coffernils M, Leon M, Gris P, Vincent JL. Blood lactate levels are superior to oxygen-derived variables in predicting outcome in human septic shock. Chest. 1991;99:956-62.

58. Friedman G, Berlot G, Kahn RJ, Vincent JL. Combined measurements of blood lactate concentrations and gastric intramucosal $\mathrm{pH}$ in patients with severe sepsis. Crit Care Med. 1995;23:1184-93.

59. Bernardin G, Pradier C, Tiger F, Deloffre P, Mattei M. Blood pressure and arterial lactate level are early indicators of short-term survival in human septic shock. Intensive Care Med. 1996;22:17-25.

60. Marecaux G, Pinsky MR, Dupont E, Kahn RJ, Vincent JL. Blood lactate levels are better prognostic indicators than TNF and IL-6 levels in patients with septic shock. Intensive Care Med. 1996;22:404-8.

61. Bakker J, Gris P, Coffernils M, Kahn RJ, Vincent JL. Serial blood lactate levels can predict the development of multiple organ failure following septic shock. Am J Surg. 1996;171:221-6.

62. Kobayashi S, Gando S, Morimoto Y, Nanzaki S, Kemmotsu O. Serial measurement of arterial lactate concentrations as a prognostic indicator in relation to the incidence of disseminated intravascular coagulation in patients with systemic inflammatory response syndrome. Surg Today. 2001;31:853-9.

63. Nguyen HB, Rivers EP, Knoblich BP, Jacobsen G, Muzzin A, Ressler JA, et al. Early lactate clearance is associated with improved outcome in severe sepsis and septic shock. Crit Care Med. 2004;32:1637-42.

64. Nguyen HB, Corbett SW, Steele R, Banta J, Clark RT, Hayes SR, et al. Implementation of a bundle of quality indicators for the early management of severe sepsis and septic shock is associated with decreased mortality. Crit Care Med. 2007;35:1105-12.

65. Phua J, Koay ES, Lee KH. Lactate, procalcitonin, and amino-terminal pro-Btype natriuretic peptide versus cytokine measurements and clinical severity scores for prognostication in septic shock. Shock. 2008;29:328-33.

66. Yang CS, Qiu HB, Huang YZ, Xie JF, Mo M, Liu SQ, et al. Prospective research on the prognosis of septic shock based on the change of lactate concentration in arterial blood. Zhonghua Wai Ke Za Zhi. 2009;47:685-8.

67. Arnold RC, Shapiro NI, Jones AE, Schorr C, Pope J, Casner E, et al. Multicenter study of early lactate clearance as a determinant of survival in patients with presumed sepsis. Shock. 2009;32:35-9.

68. Nguyen HB, Loomba M, Yang JJ, Jacobsen G, Shah K, Otero RM, et al. Early lactate clearance is associated with biomarkers of inflammation, coagulation, apoptosis, organ dysfunction and mortality in severe sepsis and septic shock. J Inflamm (Lond). 2010;7:6.

69. Puskarich MA, Trzeciak S, Shapiro NI, Arnold RC, Heffner AC, Kline JA, et al. Prognostic value and agreement of achieving lactate clearance or central venous oxygen saturation goals during early sepsis resuscitation. Acad Emerg Med. 2012;19:252-8.

70. Zanaty OM, Megahed M, Demerdash $\mathrm{H}$, Swelem R. Delta neutrophil index versus lactate clearance: early markers for outcome prediction in septic shock patients. Alex J Med. 2012;48:327-33.
71. Puskarich MA, Trzeciak S, Shapiro NI, Albers AB, Heffner AC, Kline JA, et al. Whole blood lactate kinetics in patients undergoing quantitative resuscitation for severe sepsis and septic shock. Chest. 2013;143:1548-53.

72. Walker CA, Griffith DM, Gray AJ, Datta D, Hay AW. Early lactate clearance in septic patients with elevated lactate levels admitted from the emergency department to intensive care: time to aim higher? J Crit Care. 2013;28:832-7.

73. Liu V, Morehouse JW, Soule J, Whippy A, Escobar GJ. Fluid volume, lactate values, and mortality in sepsis patients with intermediate lactate values. Ann Am Thorac Soc. 2013;10:466-73.

74. Marty P, Roquilly A, Vallee F, Luzi A, Ferre F, Fourcade O, et al. Lactate clearance for death prediction in severe sepsis or septic shock patients during the first 24 hrs in intensive care unit: an observational study. Ann Intensive Care. 2013;3:3.

75. Park JH, Lee J, Park YS, Lee CH, Lee SM, Yim JJ, et al. Prognostic value of central venous oxygen saturation and blood lactate levels measured simultaneously in the same patients with severe systemic inflammatory response syndrome and severe sepsis. Lung. 2014;192:435-40.

76. Permpikul C, Sringam P, Tongyoo S. Therapeutic goal achievements during severe sepsis and septic shock resuscitation and their association with patients' outcomes. J Med Assoc Thai. 2014;97 Suppl 3:S176-83.

77. Bao L, Zhang M, Yan P, Wu X, Shao J, Zheng R. Retrospective analysis of the value of arterial blood lactate level and its clearance rate on the prognosis of septic shock patients. Zhonghua Wei Zhong Bing Ji Jiu Yi Xue. 2015;27:38-42.

78. Galbois A, Bige N, Pichereau C, Boelle PY, Baudel JL, Bourcier S, et al. Exploration of skin perfusion in cirrhotic patients with septic shock. J Hepatol. 2015;62:549-55.

79. Lee SM, Kim SE, Kim EB, Jeong HJ, Son YK, An WS. Lactate clearance and vasopressor seem to be predictors for mortality in severe sepsis patients with lactic acidosis supplementing sodium bicarbonate: a retrospective analysis. PLoS One. 2015;10:e0145181.

80. Lokhandwala S, Moskowitz A, Lawniczak R, Giberson T, Cocchi MN, Donnino MW. Disease heterogeneity and risk stratification in sepsis-related occult hypoperfusion: a retrospective cohort study. J Crit Care. 2015;30:531-6.

81. Wang H, Li Z, Yin M, Chen XM, Ding SF, Li C, et al. Combination of Acute Physiology and Chronic Health Evaluation II score, early lactate area, and Nterminal prohormone of brain natriuretic peptide levels as a predictor of mortality in geriatric patients with septic shock. J Crit Care. 2015;30:304-9.

82. Bhat SR, Swenson KE, Francis MW, Wira CR. Lactate clearance predicts survival among patients in the emergency department with severe sepsis. West J Emerg Med. 2015;16:1118-26.

83. Chertoff J, Chisum M, Simmons L, King B, Walker M, Lascano J. Prognostic utility of plasma lactate measured between 24 and $48 \mathrm{~h}$ after initiation of early goal-directed therapy in the management of sepsis, severe sepsis, and septic shock. J Intensive Care. 2016;4:13

84. Drumheller BC, Agarwal A, Mikkelsen ME, Sante SC, Weber AL, Goyal M, et al. Risk factors for mortality despite early protocolized resuscitation for severe sepsis and septic shock in the emergency department. J Crit Care. 2016;31:13-20.

85. He HW, Liu DW, Long Y, Wang XT. High central venous-to-arterial CO2 difference/arterial-central venous $\mathrm{O} 2$ difference ratio is associated with poor lactate clearance in septic patients after resuscitation. J Crit Care. 2016;31:76-81.

86. Ha TS, Shin TG, Jo IJ, Hwang SY, Chung CR, Suh GY et al. Lactate clearance and mortality in septic patients with hepatic dysfunction. Am J Emerg Med. 2016;34:1011-15.

87. Bolvardi E, Malmir J, Reihani H, Hashemian AM, Bahramian M, Khademhosseini $P$, et al. The role of lactate clearance as a predictor of organ dysfunction and mortality in patients with severe sepsis. Mater Sociomed. 2016;28:57-60.

88. Amir A, Saulters KJ, Olum S, Pitts K, Parsons A, Churchill C et al. Outcomes of patients with severe sepsis after the first 6 hrs of resuscitation at a regional referral hospital in Uganda. J Crit Care. 2016;33:78-83.

89. Tian HH, Han SS, Lv CJ, Wang T, Li Z, Hao D, et al. The effect of early goal lactate clearance rate on the outcome of septic shock patients with severe pneumonia. Zhongguo Wei Zhong Bing Ji Jiu Yi Xue. 2012;24:42-5.

90. Yu B, Tian HY, Hu ZJ, Zhao C, Liu LX, Zhang Y, et al. Comparison of the effect of fluid resuscitation as guided either by lactate clearance rate or by central venous oxygen saturation in patients with sepsis. Zhonghua Wei Zhong Bing Ji Jiu Yi Xue. 2013;25:578-83.

91. Lyu X, Xu Q, Cai G, Yan J, Yan M. Efficacies of fluid resuscitation as guided by lactate clearance rate and central venous oxygen saturation in patients with septic shock. Zhonghua Yi Xue Za Zhi. 2015;95:496-500. 
92. Kuan WS, Ibrahim I, Leong BS, Jain S, Lu Q, Cheung YB, et al. Emergency department management of sepsis patients: a randomized, goal-oriented, noninvasive sepsis trial. Ann Emerg Med. 2016;67:367-78.

93. Attana P, Lazzeri C, Chiostri M, Picariello C, Gensini GF, Valente S. Lactate clearance in cardiogenic shock following ST elevation myocardial infarction: a pilot study. Acute Card Care. 2012:14:20-6.

94. Attana P, Lazzeri C, Chiostri M, Picariello C, Gensini GF, Valente S. Strong-ion gap approach in patients with cardiogenic shock following ST-elevation myocardial infarction. Acute Card Care. 2013;15:58-62.

95. Park TK, Yang JH, Choi SH, Song YB, Hahn JY, Choi JH, et al. Clinical outcomes of patients with acute myocardial infarction complicated by severe refractory cardiogenic shock assisted with percutaneous cardiopulmonary support. Yonsei Med J. 2014;55:920-7.

96. Guenther S, Theiss HD, Fischer M, Sattler S, Peterss S, Born F, et al. Percutaneous extracorporeal life support for patients in therapy refractory cardiogenic shock: initial results of an interdisciplinary team. Interact Cardiovasc Thorac Surg. 2014;18:283-91.

97. Kliegel A, Losert H, Sterz F, Holzer M, Zeiner A, Havel C, et al. Serial lactate determinations for prediction of outcome after cardiac arrest. Medicine (Baltimore). 2004;83:274-9.

98. Donnino MW, Miller J, Goyal N, Loomba M, Sankey SS, Dolcourt B, et al. Effective lactate clearance is associated with improved outcome in post-cardiac arrest patients. Resuscitation. 2007;75:229-34.

99. Arnalich F, Menendez M, Lagos V, Ciria E, Quesada A, Codoceo R, et al. Prognostic value of cell-free plasma DNA in patients with cardiac arrest outside the hospital: an observational cohort study. Crit Care. 2010;14:R47.

100. Le Guen M, Nicolas-Robin A, Carreira S, Raux M, Leprince P, Riou B, et al. Extracorporeal life support following out-of-hospital refractory cardiac arrest. Crit Care. 2011;15:R29.

101. Starodub R, Abella BS, Grossestreuer AV, Shofer FS, Perman SM, Leary M, et al. Association of serum lactate and survival outcomes in patients undergoing therapeutic hypothermia after cardiac arrest. Resuscitation. 2013;84:1078-82.

102. Donnino MW, Andersen LW, Giberson T, Gaieski DF, Abella BS, Peberdy MA, et al. Initial lactate and lactate change in post-cardiac arrest: a multicenter validation study. Crit Care Med. 2014:42:1804-11.

103. Riveiro DF, de Oliveira VM, Braunner JS, Vieira SR. Evaluation of serum lactate, central venous saturation, and venous-arterial carbon dioxide difference in the prediction of mortality in postcardiac arrest syndrome. J Intensive Care Med. 2015. [Epub ahead of print]

104. Williams TA, Martin R, Celenza A, Bremner A, Fatovich D, Krause J, et al. Use of serum lactate levels to predict survival for patients with out-of-hospital cardiac arrest: a cohort study. Emerg Med Australas. 2016;28:171-8.

105. Zhao YF, Lin Y, Zhu XL. Clinical significance of early lactate clearance rate in patients with respiratory failure. Zhonghua Jie He He Hu Xi Za Zhi. 2010;33:183-7

106. Wu WH, Niu YY, Zhang CR, Xiao LB, Ye HS, Pan DM, et al. Combined APACH II score and arterial blood lactate clearance rate to predict the prognosis of ARDS patients. Asian Pac J Trop Med. 2012;5:656-60.

107. Zang Z, Xu H, Dong L, Gao F, Yan J. Prognostic significance of early lactate clearance rate for severe acute respiratory failure patients on extracorporeal membrane oxygenation. Zhonghua Jie He He Hu Xi Za Zhi. 2014;37:197-201.

108. Liu XW, Ma T, Qu B, Ji Y, Liu Z. Prognostic value of initial arterial lactate level and lactate metabolic clearance rate in patients with acute paraquat poisoning. Am J Emerg Med. 2013;31:1230-5.

109. Wu JF, Wu RY, Chen J, Ou-Yang B, Chen MY, Guan XD. Early lactate clearance as a reliable predictor of initial poor graft function after orthotopic liver transplantation. Hepatobiliary Pancreat Dis Int. 2011;10:587-92.

110. Scott S, Antonaglia V, Guiotto G, Paladino F, Schiraldi F. Two-hr lactate clearance predicts negative outcome in patients with cardiorespiratory insufficiency. Crit Care Res Pract. 2010;2010:917053.

111. Mohamed KAE, Ahmed DAE. Prognostic value of lactate clearance in severe community-acquired pneumonia. Egypt J Chest Dis Tuberc. 2014;63:1053-8.

112. Lee TR, Kang MJ, Cha WC, Shin TG, Sim MS, Jo IJ, et al. Better lactate clearance associated with good neurologic outcome in survivors who treated with therapeutic hypothermia after out-of-hospital cardiac arrest. Crit Care. 2013;17:R260

113. Matsumoto $H$, Nihei $S$, Endo T, Kanazawa A, Arai $H$, Nagata $K$, et al. Examination of relationship between lactate clearance and neurologic outcome in cardiac arrest induced by ventricular fibrillation. J UOEH. 2014;36:11-6.
114. Qvisth V, Hagstrom-Toft E, Enoksson S, Bolinder J. Catecholamine regulation of local lactate production in vivo in skeletal muscle and adipose tissue: role of -adrenoreceptor subtypes. J Clin Endocrinol Metab. 2008;93:240-6.

115. Contenti J, Occelli C, Corraze H, Lemoel F, Levraut J. Long-term beta-blocker therapy decreases blood lactate concentration in severely septic patients. Crit Care Med. 2015;43:2616-22.

116. Orbegozo Cortes D, Rayo Bonor A, Vincent JL. Isotonic crystalloid solutions: a structured review of the literature. Br J Anaesth. 2014;112:968-81.

117. Didwania A, Miller J, Kassel D, Jackson Jr EV, Chernow B. Effect of intravenous lactated Ringer's solution infusion on the circulating lactate concentration: Part 3. Results of a prospective, randomized, double-blind, placebo-controlled trial. Crit Care Med. 1997;25:1851-4

118. Dezman ZD, Comer AC, Narayan M, Scalea TM, Hirshon JM, Smith GS Alcohol consumption decreases lactate clearance in acutely injured patients. Injury. 2016. [Epub ahead of print]

119. Kruse JA, Zaidi SA, Carlson RW. Significance of blood lactate levels in critically ill patients with liver disease. Am J Med. 1987;83:77-82.

120. Chiolero R, Tappy L, Gillet M, Revelly JP, Roth H, Cayeux C, et al. Effect of major hepatectomy on glucose and lactate metabolism. Ann Surg. 1999;229:505-13.

121. Tapia P, Soto D, Bruhn A, Alegria L, Jarufe N, Luengo C, et al. Impairment of exogenous lactate clearance in experimental hyperdynamic septic shock is not related to total liver hypoperfusion. Crit Care. 2015;19:188.

122. Schmidt LE, Larsen FS. Prognostic implications of hyperlactatemia, multiple organ failure, and systemic inflammatory response syndrome in patients with acetaminophen-induced acute liver failure. Crit Care Med. 2006;34:337-43.

123. Hernandez G, Luengo C, Bruhn A, Kattan E, Friedman G, Ospina-Tascon GA, et al. When to stop septic shock resuscitation: clues from a dynamic perfusion monitoring. Ann Intensive Care. 2014;4:30.

\section{Submit your next manuscript to BioMed Central and we will help you at every step:}

- We accept pre-submission inquiries

- Our selector tool helps you to find the most relevant journal

- We provide round the clock customer support

- Convenient online submission

- Thorough peer review

- Inclusion in PubMed and all major indexing services

- Maximum visibility for your research

Submit your manuscript at www.biomedcentral.com/submit
BioMed Central 